\title{
The Dynamic Behavior of a Class of Kirchhoff Equations with High Order Strong Damping
}

\author{
Guoguang Lin, Chunmeng Zhou* \\ Department of Mathematics, Yunnan University, Kunming, China \\ Email: *gglin@ynu.edu.cn, ${ }^{\star 9} 954137301 @ q q . c o m$
}

How to cite this paper: Lin, G.G. and Zhou, C.M. (2021) The Dynamic Behavior of a Class of Kirchhoff Equations with High Order Strong Damping. Journal of Applied Mathematics and Physics, 9, 1041-1055. https://doi.org/10.4236/jamp.2021.95071

Received: April 19, 2021

Accepted: May 23, 2021

Published: May 26, 2021

Copyright $\odot 2021$ by author(s) and Scientific Research Publishing Inc. This work is licensed under the Creative Commons Attribution International License (CC BY 4.0).

http://creativecommons.org/licenses/by/4.0/

(c) (i) Open Access

\begin{abstract}
In this paper, we study the long time behavior of a class of Kirchhoff equations with high order strong dissipative terms. On the basis of the proper hypothesis of rigid term and nonlinear term of Kirchhoff equation, firstly, we evaluate the equation via prior estimate in the space $E_{0}$ and $E_{k}$, and verify the existence and uniqueness of the solution of the equation by using Galerkin's method. Then, we obtain the bounded absorptive set $B_{0 k}$ on the basis of the prior estimate. Moreover, by using the Rellich-Kondrachov Compact Embedding theorem, we prove that the solution semigroup $S(t)$ of the equation has the family of the global attractor $A_{k}$ in space $E_{k}$. Finally, we prove that the solution semigroup $S(t)$ is Frechet differentiable on $E_{k}$ via linearizing the equation. Furthermore, we can obtain the finite Hausdorff dimension and Fractal dimension of the family of the global attractor $A_{k}$.
\end{abstract}

\section{Keywords}

Kirchhoff Equation, Prior Estimate, The Existence and Uniqueness of the Solution, Family of Global Attractor, Dimension Estimation

\section{Introduction}

In this paper, we mainly study the initial boundary value problem of the Kirchhoff equation with high order strong damping:

$$
\begin{aligned}
& u_{t t}+M\left(\left\|\nabla^{m} u\right\|_{p}^{p}\right)(-\Delta)^{2 m} u+\beta(-\Delta)^{2 m} u_{t}+|u|^{\rho} u=f(x), \\
& u(x, t)=\frac{\partial^{j} u}{\partial v^{j}}=0, \quad j=1,2, \cdots, 2 m-1, \quad x \in \partial \Omega, \\
& u(x, 0)=u_{0}(x), u_{t}(x, 0)=u_{1}(x), \quad x \in \partial \Omega, \quad t>0,
\end{aligned}
$$

where $m>1, p \geq 2, \Omega \subseteq R^{n}(n \geq 1)$ is a bounded region with a smooth boun- 
dary $\partial \Omega, Q=\Omega \times[0, \infty)$ stands for the cylinder in $R_{x}^{n} \times R_{t}$, the rigid term $M(s) \in C^{1}[0, \infty)$ is a general function, $\beta(-\Delta)^{2 m} u_{t}(\beta>0)$ is the strong dissipative term, $|u|^{\rho} u$ is the nonlinear term and $\rho \geq-1, f(x)$ denotes the external force.

Kirchhoff equation is a kind of important nonlinear wave equation, which is widely used in engineering physics, especially provides a strong support for measuring bridge vibration. Kirchhoff equation originates from a physical model, which is obtained by German physicist Gustav Robert Kirchhoff [1] when he studied the transverse vibration of elastic string:

$$
\rho h \frac{\partial^{2} u}{\partial t^{2}}-\left[P_{0}+\frac{E h}{2 L} \int_{0}^{L}\left(\frac{\partial u}{\partial x}\right)^{2} \mathrm{~d} x\right] \frac{\partial^{2} u}{\partial x^{2}}=f, \quad 0<x<L, t \geq 0,
$$

As $m=1, p=2, \beta=1$, Masanori et al. studied Kirchhoff equations with nonlinear dissipative terms in the literature [2]:

$$
u_{t t}-M\left(\|\nabla u\|^{2}\right) \Delta u+\delta|u|^{\rho} u+r u_{t}=f,
$$

and they had discussed the existence and attenuation of the global solution of the initial boundary value problem by using Galerkin's method.

Then Igor Chueshov studied the Kirchhoff equation with strong dissipative term for $m=1, p=2, \beta=\sigma\left(\|\nabla u\|^{2}\right)$ in reference [3]:

$$
u_{t t}-\sigma\left(\|\nabla u\|^{2}\right) \Delta u_{t}-\varphi\left(\|\nabla u\|^{2}\right) \Delta u+g(u)=f(x),
$$

They proved that its weak solution exists and is unique. Particularly, the equation has strong exponential attractor when the nonlinear term $g(u)$ is in a non-supercritical state. Further, Guoguang Lin, Yuhang Chen [4] et al. extended the equation to a higher order Kirchhoff-type equation based on the study of Igor Chueshov, and added a structural dissipation term $\Delta^{2 m} u$ :

$$
u_{t t}+N\left(\left\|\nabla^{m} u\right\|_{q}^{q}\right)(-\Delta)^{m} u+a(t)(-\Delta)^{m} u_{t}+\Delta^{2 m} u=I(x),
$$

They discussed the relationship between the order $m$ and $q$, made reasonable assumptions about the relevant terms, proved the existence and uniqueness of the comprehensions, and made finite-dimensional estimates of the global attractors. When the coefficient of structural dissipation term is not equal to 1 , they can't get the relevant conclusion. More research results can be referred to [5]-[16].

On the basis of previous studies, we improve the order of strong dissipation term further in this paper. At the same time, summing up previous experience, we discuss the difficult problem of the relationship between order $m$ and $p$ in the rigid term and $\rho$ in the nonlinear term, and get some theoretical results about the long time behavior of the equation.

\section{The Basic Assumptions}

For the convenience of later narration, the space and sign mentioned in the ar- 
ticle are defined as follows:

$$
H=L^{2}(\Omega), \quad H_{0}^{m}(\Omega)=H^{m}(\Omega) \cap H_{0}^{1}(\Omega), \quad E_{k}=H_{0}^{2 m+k}(\Omega) \times H_{0}^{k}(\Omega), \quad A^{k} \quad \text { is }
$$

the global attractor family from $E_{0}$ to $E_{k}, B_{0 k}$ is a bounded absorption set in $E_{0}$, where $k=1,2, \cdots, 2 m, C_{i}>0(i=0,1,2, \cdots)$ are constants. The inner product $(\cdot, \cdot)$ and norm $\|\cdot\|$ of $H$ are given by $(u, v)=\int_{\Omega} u(x) v(x) \mathrm{d} x,\|\cdot\|=\|\cdot\|_{L^{2}(\Omega)}$, $\|\cdot\|_{p}=\|\cdot\|_{L^{p}(\Omega)}$.

$M(s), \rho$ and $p$ satisfy the following conditions:

(A1) For $\forall s \geq 0$, we have $\varepsilon+1 \leq \mu_{0} \leq M(s) \leq \mu_{1}$, where $\mu_{0}, \mu_{1}$ are constants, and

$$
\mu= \begin{cases}\mu_{0}, & \frac{\mathrm{d}}{\mathrm{d} t}\left\|\Delta^{m} u\right\|^{2} \geq 0 \\ \mu_{1}, & \frac{\mathrm{d}}{\mathrm{d} t}\left\|\Delta^{m} u\right\|^{2} \leq 0\end{cases}
$$

(A2) $\left\{\begin{array}{cc}1 \leq \rho \leq \frac{8 m}{n-4 m}, & n>4 m \\ \rho \geq-1, & n \leq 4 m\end{array} ;\right.$

(A3) $\left\{\begin{array}{cc}2 \leq p \leq \frac{2 n}{n-2 m}, & n>2 m \\ p \geq 2, & n \leq 2 m\end{array} ;\right.$

(A4) $0 \leq \varepsilon \leq \min \left\{\sqrt{1+\frac{\beta \lambda_{1}^{2 m}}{4}-1}, \frac{2 \mu}{\beta}, \frac{2 \mu \lambda_{1}^{2 m}}{\beta \lambda_{1}^{2 m}+1}\right\} ; \quad \lambda_{1}$ denotes the first eigenvalue of $-\Delta$ with the homogeneous Dirichlet boundary on $\Omega$.

\section{Existence and Uniqueness of Solutions}

Lemma 1. Assume (A1)-(A4) are valid. Let $\left(u_{0}, u_{1}\right) \in E_{0}, f(x) \in L^{2}(\Omega)$, then the initial boundary value problem $(1.1)$ has a global solution $(u, v)$ that satisfies $u \in L^{\infty}\left(0,+\infty ; H_{0}^{2 m}(\Omega)\right)$,

$$
\begin{gathered}
v \in L^{\infty}\left(0,+\infty ; L^{2}(\Omega)\right) \cap L^{2}\left(0, T ; H_{0}^{2 m}(\Omega)\right), \text { and } \\
\|(u, v)\|_{E_{0}}^{2}=\left\|\Delta^{m} u\right\|^{2}+\|v\|^{2} \leq y_{1}(0) \mathrm{e}^{-\alpha_{1} t}+\frac{C_{1}}{\alpha_{1}}, \\
\frac{\beta}{2} \int_{0}^{T}\left\|\Delta^{m} v\right\|^{2} \mathrm{~d} t \leq C_{1} T+y_{1}(0),
\end{gathered}
$$

where $v=u_{t}+\varepsilon u, \quad \alpha_{1}=\min \left\{\frac{\beta \lambda_{1}^{2 m}}{2}-2 \varepsilon-\varepsilon^{2}, 2 \varepsilon, \frac{2 \varepsilon \mu-\beta \varepsilon^{2}}{\mu}, \varepsilon(\rho+2)\right\}$, $y_{1}(0)=\left\|v_{0}\right\|^{2}+\varepsilon^{2}\left\|u_{0}\right\|^{2}+\mu\left\|\Delta^{m} u_{0}\right\|^{2}+\frac{2}{\rho+2}\left\|u_{0}\right\|_{\rho+2}^{\rho+2}$.

So there exists a nonnegative real number $R_{1}$ and $t_{1}=t_{1}(\Omega)>0$ such that

$$
\|(u, v)\|_{E_{0}}^{2}=\left\|\Delta^{m} u\right\|^{2}+\|v\|^{2} \leq R_{1}^{2} \quad\left(t>t_{1}\right)
$$

Proof. Let $v=u_{t}+\varepsilon u$, take the inner product of Equation (1.1) with $v$ in $H$, and we get that 


$$
\left(u_{t t}+M\left(\left\|D^{m} u\right\|_{p}^{p}\right)(-\Delta)^{2 m} u+\beta(-\Delta)^{2 m} u_{t}+|u|^{\rho} u, v\right)=(f(x), v) .
$$

We process the terms in Equation (3.4) by using Young's inequality, Holder's inequality, Poincare's inequality and differential mean value theorem, then we obtain that

$$
\begin{gathered}
\left(u_{t t}, v\right)=\frac{1}{2} \frac{\mathrm{d}}{\mathrm{d} t}\|v\|^{2}+\frac{\varepsilon^{2}}{2}\|u\|^{2}-\varepsilon\|v\|^{2}+\varepsilon^{3}\|u\|^{2} . \\
\left(M\left(\left\|D^{m} u\right\|_{p}^{p}\right)(-\Delta)^{2 m} u, v\right) \geq \frac{\mu}{2} \frac{\mathrm{d}}{\mathrm{d} t}\left\|\Delta^{m} u\right\|^{2}+\varepsilon \mu\left\|\Delta^{m} u\right\|^{2}, \\
\left(\beta(-\Delta)^{2 m} u_{t}, v\right) \geq \frac{\beta}{4}\left\|\Delta^{m} v\right\|^{2}+\frac{\beta \lambda_{1}^{2 m}}{4}\|v\|^{2}-\frac{\beta \varepsilon^{2}}{2}\left\|\Delta^{m} u\right\|^{2}, \\
\left(|u|^{\rho} u, v\right)=\frac{1}{\rho+2} \frac{\mathrm{d}}{\mathrm{d} t}\|u\|_{\rho+2}^{\rho+2}+\varepsilon\|u\|_{\rho+2}^{\rho+2}, \\
(f(x), v) \leq \frac{1}{2 \varepsilon^{2}}\|f\|^{2}+\frac{\varepsilon^{2}}{2}\|v\|^{2} .
\end{gathered}
$$

Substitute (3.5)-(3.9) into (3.4), we have

$$
\begin{gathered}
\frac{\mathrm{d}}{\mathrm{d} t}\left(\|v\|^{2}+\varepsilon^{2}\|u\|^{2}+\mu\left\|\Delta^{m} u\right\|^{2}+\frac{2}{\rho+2}\|u\|_{\rho+2}^{\rho+2}\right)+\left(\frac{\beta \lambda_{1}^{2 m}}{2}-2 \varepsilon-\varepsilon^{2}\right)\|v\|^{2} \\
+2 \varepsilon^{3}\|u\|^{2}+\left(2 \varepsilon \mu-\beta \varepsilon^{2}\right)\left\|\Delta^{m} u\right\|^{2}+2 \varepsilon\|u\|_{\rho+2}^{\rho+2}+\frac{\beta}{2}\left\|\Delta^{m} v\right\|^{2} \leq \frac{1}{\varepsilon^{2}}\|f\|^{2}=C_{1} . \\
\text { Let } \alpha_{1}=\min \left\{\frac{\beta \lambda_{1}^{2 m}}{2}-2 \varepsilon-\varepsilon^{2}, 2 \varepsilon, \frac{2 \varepsilon \mu-\beta \varepsilon^{2}}{\mu}, \varepsilon(\rho+2)\right\} \text {, then } \\
\frac{\mathrm{d} y_{1}(t)}{\mathrm{d} t}+\alpha_{1} y_{1}(t)+\frac{\beta}{2}\left\|\Delta^{m} v\right\|^{2} \leq C_{1},
\end{gathered}
$$

where $y_{1}(t)=\|v\|^{2}+\varepsilon^{2}\|u\|^{2}+\mu\left\|\Delta^{m} u\right\|^{2}+\frac{2}{\rho+2}\|u\|_{\rho+2}^{\rho+2}$.

By using the differential Gronwall's inequality, we obtain

$$
\begin{gathered}
y_{1}(t) \leq y_{1}(0) \mathrm{e}^{-\alpha_{1} t}+\frac{C_{1}}{\alpha_{1}}, \\
\frac{\beta}{2} \int_{0}^{T}\left\|\Delta^{m} v\right\|^{2} \mathrm{~d} t \leq C_{1} T+y_{1}(0),
\end{gathered}
$$

thus $\|(u, v)\|_{E_{0}}^{2}=\left\|\Delta^{m} u\right\|^{2}+\|v\|^{2} \leq y_{1}(t)$, moreover

$$
\varlimsup_{t \rightarrow+\infty}\|(u, v)\|_{E_{0}}^{2} \leqslant \frac{C_{1}}{\alpha_{1}}=R_{1}^{2} .
$$

Lemma 1 is proved.

Lemma 2. Assume (A1)-(A4) are valid. If $\left(u_{0}, u_{1}\right) \in E_{k}, k=1,2, \cdots, 2 m$, $f(x) \in L^{2}(\Omega)$, then the initial boundary value problem (1) has a global solution $(u, v)$ that satisfies $u \in L^{\infty}\left(0,+\infty ; H_{0}^{2 m+k}(\Omega)\right)$, $v \in L^{\infty}\left(0,+\infty ; H_{0}^{k}(\Omega)\right) \cap L^{2}\left(0, T ; H_{0}^{2 m+k}(\Omega)\right)$, and 


$$
\begin{gathered}
\|(u, v)\|_{E_{k}}^{2}=\left\|\nabla^{2 m+k} u\right\|^{2}+\left\|\nabla^{k} v\right\|^{2} \leq y_{2}(0) e^{-\alpha_{2} t}+\frac{C_{3}}{\alpha_{2}}, \\
\frac{\beta}{4} \int_{0}^{T}\left\|\nabla^{2 m+k} v\right\|^{2} \mathrm{~d} t \leq C_{3} T+y_{2}(0),
\end{gathered}
$$

where $v=u_{t}+\varepsilon u, \quad \alpha_{2}=\min \left\{\frac{\beta \lambda_{1}^{2 m}}{4}-2 \varepsilon-\varepsilon^{2}, 2 \varepsilon-\frac{\varepsilon^{2}}{\lambda_{1}^{2 m} \mu}-\frac{\beta \varepsilon^{2}}{\mu}\right\}$,

$y_{2}(0)=\left\|\nabla^{k} v_{0}\right\|^{2}+\mu\left\|\nabla^{2 m+k} u_{0}\right\|^{2}$.

Thus there exists a nonnegative real number $R_{k}$ and $t_{2}=t_{2}(\Omega)>0$ such that

$$
\|(u, v)\|_{E_{k}}^{2}=\left\|\nabla^{2 m+k} u\right\|^{2}+\left\|\nabla^{k} v\right\|^{2} \leq R_{k}^{2}, \quad\left(t>t_{2}\right) .
$$

Proof. Taking the inner product of Equation (1.1) with $(-\Delta)^{k} v$ in $H$, and we get that

$\left(u_{t t}+M\left(\left\|D^{m} u\right\|_{p}^{p}\right)(-\Delta)^{2 m} u+\beta(-\Delta)^{2 m} u_{t}+|u|^{\rho} u,(-\Delta)^{k} v\right)=\left(f(x),(-\Delta)^{k} v\right)$.

Similar to Lemma 1, each item of Equation (3.12) can be obtained

$$
\begin{gathered}
\left(u_{t t},(-\Delta)^{k} v\right) \geq \frac{1}{2} \frac{\mathrm{d}}{\mathrm{d} t}\left\|\nabla^{k} v\right\|^{2}-\frac{2 \varepsilon+\varepsilon^{2}}{2}\left\|\nabla^{k} v\right\|^{2}-\frac{\varepsilon^{2}}{2 \lambda_{1}^{2 m}}\left\|\nabla^{2 m+k} u\right\|^{2} \\
\left(M\left(\left\|D^{m} u\right\|_{p}^{p}\right)(-\Delta)^{2 m} u,(-\Delta)^{k} v\right) \geq \frac{\mu}{2} \frac{\mathrm{d}}{\mathrm{d} t}\left\|\nabla^{2 m+k} u\right\|^{2}+\varepsilon \mu\left\|\nabla^{2 m+k} u\right\|^{2} \\
\left(\beta(-\Delta)^{2 m} u_{t},(-\Delta)^{k} v\right) \\
\geq \frac{\beta}{8}\left\|\nabla^{2 m+k} v\right\|^{2}-\frac{\beta \varepsilon^{2}}{2}\left\|\nabla^{2 m+k} u\right\|^{2}+\frac{\beta \lambda_{1}^{2 m-k}}{4}\left\|(-\Delta)^{k} v\right\|^{2}+\frac{\beta \lambda_{1}^{2 m}}{8}\left\|\nabla^{k} v\right\|^{2}, \\
\left\|\left(|u|^{\rho} u,(-\Delta)^{k} v\right) \mid \geq-\right\| u\left\|_{\infty}^{\rho+1}\right\|(-\Delta)^{k} v\left\|\geq-\frac{2 C_{2}}{\beta \lambda_{1}^{2 m-k}}-\frac{\beta \lambda_{1}^{2 m-k}}{8}\right\|(-\Delta)^{k} v \|^{2}, \\
\left(f(x),(-\Delta)^{k} v\right) \leq \frac{2}{\beta \lambda_{1}^{2 m-k}}\|f(x)\|^{2}+\frac{\beta \lambda_{1}^{2 m-k}}{8}\left\|(-\Delta)^{k} v\right\|^{2}
\end{gathered}
$$

Substitute inequality (3.13)-(3.17) into (3.12), we have

$$
\begin{aligned}
& \frac{\mathrm{d}}{\mathrm{d} t}\left(\left\|\nabla^{k} v\right\|^{2}+\mu\left\|\nabla^{2 m+k} u\right\|^{2}\right)+\left(\frac{\beta \lambda_{1}^{2 m}}{4}-2 \varepsilon-\varepsilon^{2}\right)\left\|\nabla^{k} v\right\|^{2} \\
& +\left(2 \varepsilon \mu-\frac{\varepsilon^{2}}{\lambda_{1}^{2 m}}-\beta \varepsilon^{2}\right)\left\|\nabla^{2 m+k} u\right\|^{2}+\frac{\beta}{4}\left\|\nabla^{2 m+k} v\right\|^{2} \\
& \leq \frac{4}{\beta \lambda_{1}^{2 m-k}}\|f(x)\|^{2}+\frac{4 C_{2}}{\beta \lambda_{1}^{2 m-k}}=C_{3} .
\end{aligned}
$$

Let $\alpha_{2}=\min \left\{\frac{\beta \lambda_{1}^{2 m}}{4}-2 \varepsilon-\varepsilon^{2}, 2 \varepsilon-\frac{\varepsilon^{2}}{\lambda_{1}^{2 m} \mu}-\frac{\beta \varepsilon^{2}}{\mu}\right\}$, then

$$
\frac{\mathrm{d} y_{2}(x)}{\mathrm{d} t}+\alpha_{2} y_{2}(x)+\frac{\beta}{4}\left\|\nabla^{2 m+k} v\right\|^{2} \leq C_{3},
$$

where $y_{2}(x)=\left\|\nabla^{k} v\right\|^{2}+\mu\left\|\nabla^{2 m+k} u\right\|^{2}$. 
By using the differential Gronwall's inequality, we obtain

$$
\begin{gathered}
y_{2}(x) \leq y_{2}(0) \mathrm{e}^{-\alpha_{2} t}+\frac{C_{3}}{\alpha_{2}}, \\
\frac{\beta}{4} \int_{0}^{T}\left\|\nabla^{2 m+k} v\right\|^{2} \mathrm{~d} t \leq y_{2}(0)+C_{3} T .
\end{gathered}
$$

So $\|(u, v)\|_{E_{k}}^{2}=\left\|\nabla^{2 m+k} u\right\|^{2}+\left\|\nabla^{k} v\right\|^{2} \leq y_{2}(t)$, moreover

$$
\varlimsup_{t \rightarrow+\infty}\|(u, v)\|_{E_{k}}^{2} \leq \frac{C_{3}}{\alpha_{2}}=R_{k}^{2} .
$$

Lemma 2 is proved.

Lemma 3. (Daprato G. [9]) Let $D$ be the bounded region of $R_{x}^{n} \times R_{t}, g_{\mu}$ and $g$ are functions of $L^{q}(D)(1<q<\infty),\left\|g_{\mu}\right\|_{L^{q}(D)}<C, g_{\mu} \rightarrow g$ a.e. in $D$, then $g_{\mu} \rightarrow g$ is weak convergence in $L^{q}(D)$.

Theorem 1. Assume (A1)-(A4) are valid. If $f \in H,\left(u_{0}, u_{1}\right) \in E_{k}$, then the initial boundary value problem (1.1) exists a unique smooth solution $(u, v) \in L^{\infty}\left([0,+\infty) ; E_{k}\right)$.

Proof. First of all, we prove the existence of solution.

Let $(-\Delta)^{2 m+k} \omega_{j}=\lambda_{j}^{2 m+k} \omega_{j}(k=1,2, \cdots, 2 m)$, where $\lambda_{j}$ represents the eigenvalue of $-\Delta$ with the homogeneous Dirichlet boundary on $\Omega, \omega_{j}$ represents the eigenfunction determined by the corresponding eigenvalue $\lambda_{j}$, then $\omega_{1}, \omega_{2}, \cdots, \omega_{h}$ constitute the standard orthonormal basis of $H$. Suppose $u_{h}=u_{h}(t)=\sum_{j=1}^{h} g_{j h}(t) \omega_{j}$ is the approximate solution of the initial boundary value problem $(1.1)$, where $g_{j h}(t)(1 \leq j \leq h)$ is determined by

$$
\begin{aligned}
& \left(u_{h}^{\prime \prime}+M\left(\left\|D^{m} u_{h}\right\|_{p}^{p}\right)(-\Delta)^{2 m} u_{h}+\beta(-\Delta)^{2 m} u_{h}^{\prime}+\left|u_{h}\right|^{\rho} u_{h},(-\Delta)^{k} \omega_{j}\right) \\
& =\left(f(x),(-\Delta)^{k} \omega_{j}\right) \quad(j=1,2, \cdots, h)
\end{aligned}
$$

Equation (3.19) satisfies the initial conditions

$$
\begin{gathered}
u_{h}(0)=u_{0 h}=\sum_{j=1}^{h} \alpha_{j h}(t) \omega_{j} \rightarrow u_{0}(h \rightarrow+\infty) \text { in } H_{0}^{2 m+k}, \\
u_{h}^{\prime}(0)=u_{1 h}=\sum_{j=1}^{h} \beta_{j h}(t) \omega_{j} \rightarrow u_{1}(h \rightarrow+\infty) \text { in } H_{0}^{k},
\end{gathered}
$$

namely as $h \rightarrow+\infty,\left(u_{0 h}, u_{1 h}\right) \rightarrow\left(u_{0}, u_{1}\right)$ in $E_{k}$. According to the basic theory of solutions of ordinary differential equations, the approximate solution $u_{h}(t)$ exists on $\left(0, t_{h}\right)$.

Multiply both sides of (3.19) by $g_{j h}^{\prime}+\varepsilon g_{j h}(t)$ and sum over $j$. Let $v_{h}(t)=u_{h}^{\prime}(t)+\varepsilon u_{h}(t)$, according to Lemma 1 and Lemma 2, estimation (3.3), (3.10), (3.11) still hold for $u_{h} \in H_{0}^{2 m+k}(\Omega), v_{h} \in H_{0}^{k}(\Omega)$, namely we have

$$
\begin{gathered}
\left\|\left(u_{h}, v_{h}\right)\right\|_{E_{k}}^{2}=\left\|\nabla^{2 m+k} u_{h}\right\|^{2}+\left\|\nabla^{k} v_{h}\right\|^{2} \leq R_{k}^{2}, \\
\frac{\beta}{4} \int_{0}^{T}\left\|\nabla^{2 m+k} v_{h}\right\|^{2} \mathrm{~d} t \leq y_{2}(0)+\int_{0}^{T} C_{3} \mathrm{~d} t,
\end{gathered}
$$


then we obtain $\left(u_{h}(t), u_{h}^{\prime}(t)\right)$ is uniformly bounded on $E_{k}$, and (3.20), (3.21) hold the priori estimate of the solution in the Lemma 2. Moreover, we know from (3.20) that $\left(u_{h}, v_{h}\right)$ is bounded in $L^{\infty}\left([0,+\infty) ; E_{k}\right)$, from (3.21) that $v_{h}$ is bounded in $L^{2}\left((0, T) ; H_{0}^{2 m+k}\right)$.

In space $E_{k}$, select the subsequence $\left\{u_{s}\right\}$ from the sequence $\left\{u_{h}\right\}$ such that $\left(u_{s}, v_{s}\right) \rightarrow(u, v)$ is weak * convergence in $L^{\infty}\left([0,+\infty) ; E_{k}\right)$, and we obtain that $v_{s}$ is bounded in $L^{2}\left((0, T) ; H_{0}^{2 m+k}\right)$ via (3.21).

Due to the Rellich-Kohdrachov Compact Embedding theorem, $E_{k} \hookrightarrow E_{0}$, $\left(u_{s}, v_{s}\right) \rightarrow(u, v)$ is strong convergence a.e. in $E_{0}$.

According to Lemma 3, taking $g_{s}=\left|u_{s}\right|^{\rho} u_{s}, q=\frac{\rho+2}{\rho+1}, g_{s} \rightarrow|u|^{\rho} u$ a.e. in $L^{q}(Q)$, where $Q=[0,+\infty) \times \Omega$, then

$$
\left|u_{s}\right|^{\rho} u_{s} \rightarrow|u|^{\rho} u \text { is weak * convergence in } L^{\infty}\left([0,+\infty) ; L^{q}(\Omega)\right) .
$$

Then in Equation (3.19), let $h=s$ and take the limit. For fixed $j$ and $s \geq j$, we obtain

$$
\begin{aligned}
& \left(u_{s}^{\prime \prime}+M\left(\left\|D^{m} u_{s}\right\|_{p}^{p}\right)(-\Delta)^{2 m} u_{s}+\beta(-\Delta)^{2 m} u_{s}^{\prime}+\left|u_{s}\right|^{\rho} u_{s},(-\Delta)^{k} \omega_{j}\right) \\
& =\left(f(x),(-\Delta)^{k} \omega_{j}\right) .
\end{aligned}
$$

Due to $u_{h} \rightarrow u$ is weak * convergence in $L^{\infty}\left([0,+\infty) ; H_{0}^{2 m+k}(\Omega)\right)$, we have

$$
\begin{aligned}
& \left(u_{s}(t),(-\Delta)^{k} \omega_{j}\right) \rightarrow\left(u(t), \lambda_{j}^{k} \omega_{j}\right) \text { is weak }{ }^{*} \text { convergence in } L^{\infty}[0,+\infty), \\
& \left(u_{s}^{\prime}(t),(-\Delta)^{k} \omega_{j}\right) \rightarrow\left(u^{\prime}(t), \lambda_{j}^{k} \omega_{j}\right) \text { is weak }{ }^{*} \text { convergence in } L^{\infty}[0,+\infty) .
\end{aligned}
$$

Then, we obtain $\left(u_{s}^{\prime \prime}(t),(-\Delta)^{k} \omega_{j}\right)=\frac{\mathrm{d}}{\mathrm{d} t}\left(u_{s}^{\prime}(t),(-\Delta)^{k} \omega_{j}\right) \rightarrow\left(u^{\prime \prime}(t), \lambda_{j}^{k} \omega_{j}\right)$ is convergence in $D^{\prime}[0,+\infty)$, where $D^{\prime}[0,+\infty)$ is the conjugate space of the infinitely differentiable space $D[0,+\infty)$.

$$
\left(M\left(\left\|D^{m} u_{s}\right\|_{p}^{p}\right)(-\Delta)^{2 m} u_{s},(-\Delta)^{k} \omega_{j}\right) \rightarrow\left(M\left(\left\|D^{m} u\right\|_{p}^{p}\right)(-\Delta)^{\frac{2 m+k}{2}} u, \lambda_{j}^{\frac{2 m+k}{2}}(-\Delta)^{k} \omega_{j}\right)
$$

is weak * convergence in $L^{\infty}[0,+\infty)$.

Due to

$$
\begin{aligned}
& \left(\beta(-\Delta)^{2 m} u_{s}^{\prime},(-\Delta)^{k} \omega_{j}\right) \\
& =\left(\beta(-\Delta)^{\frac{k}{2}} v_{s},(-\Delta)^{\frac{2 m+k}{2}} \omega_{j}\right)-\beta \varepsilon\left((-\Delta)^{\frac{2 m+k}{2}} u_{s},(-\Delta)^{\frac{2 m+k}{2}} \omega_{j}\right)^{\prime} \\
& \left(\beta(-\Delta)^{2 m} u_{s}^{\prime},(-\Delta)^{k} \omega_{j}\right) \\
& \rightarrow\left(\beta(-\Delta)^{\frac{k}{2}} v, \lambda_{j}(-\Delta)^{\frac{2 m+k}{2}} \omega_{j}\right)-\beta \varepsilon\left((-\Delta)^{\frac{2 m+k}{2}} u, \lambda_{j}^{\frac{2 m+k}{2}} \omega_{j}\right) \text { is }
\end{aligned}
$$

weak * convergence in $L^{\infty}[0,+\infty)$.

According to Equation (3.22), we have 
$\left(\left|u_{s}\right|^{\rho} u_{s},(-\Delta)^{k} \omega_{j}\right) \rightarrow\left(|u|^{\rho} u, \lambda_{j}^{k} \omega_{j}\right)$ is weak ${ }^{\star}$ convergence in $L^{\infty}[0,+\infty)$.

Because $\omega_{j}$ is arbitrary, for $\forall v \in H^{k}(\Omega) \cap H_{0}^{1}(\Omega)$ we have

$$
\begin{aligned}
& \left(u_{t t}+M\left(\left\|D^{m} u\right\|_{p}^{p}\right)(-\Delta)^{2 m} u+\beta(-\Delta)^{2 m} u_{t}+|u|^{\rho} u,(-\Delta)^{k} \omega_{j}\right) \\
& =\left(f(x),(-\Delta)^{k} \omega_{j}\right) .
\end{aligned}
$$

Hence, Equation (3.23) is established for all $j$. The existence is proved.

Then we prove the solution of the initial and boundary value problem is unique.

Assume $u_{1}, u_{2}$ are the solution of the initial and boundary value problem (1.1). Let $w=u_{1}-u_{2}$, we have

$$
\begin{aligned}
& w_{t t}+M\left(\left\|D^{m} u_{1}\right\|_{p}^{p}\right)(-\Delta)^{2 m} u_{1}-M\left(\left\|D^{m} u_{2}\right\|_{p}^{p}\right)(-\Delta)^{2 m} u_{2} \\
& +\beta(-\Delta)^{2 m} w_{t}+\left|u_{1}\right|^{\rho} u_{1}-\left|u_{2}\right|^{\rho} u_{2}=0, \\
& w(0)=0, w_{t}(0)=0, \\
& w \in L^{\infty}\left([0,+\infty) ; H_{0}^{2 m}(\Omega)\right), w_{t} \in L^{\infty}\left([0,+\infty) ; L^{2}(\Omega)\right) .
\end{aligned}
$$

Taking the inner product of Equation (3.24) with $w_{t}$ in $H$, and we get that

$$
\begin{aligned}
& \frac{1}{2} \frac{\mathrm{d}}{\mathrm{d} t}\left\|w_{t}\right\|^{2}+\beta\left\|\Delta^{m} w_{t}\right\|^{2}+\left(M\left(\left\|\nabla^{m} u_{1}\right\|_{p}^{p}\right)(-\Delta)^{2 m} u_{1}-M\left(\left\|\nabla^{m} u_{2}\right\|_{p}^{p}\right)(-\Delta)^{2 m} u_{2}, w_{t}\right) \\
& +\left(\left|u_{1}\right|^{\rho} u_{1}-\left|u_{2}\right|^{\rho} u_{2}, w_{t}\right)=0 .
\end{aligned}
$$

According to Lemma 1 and Lemma 2, the Equation (3.25) is processed as follows

$$
\begin{gathered}
\left(M\left(\left\|\nabla^{m} u_{1}\right\|_{p}^{p}\right)(-\Delta)^{2 m} u_{1}-M\left(\left\|\nabla^{m} u_{2}\right\|_{p}^{p}\right)(-\Delta)^{2 m} u_{2}, w_{t}\right) \\
\geq \frac{1}{2} M\left(\left\|\nabla^{m} u_{1}\right\|_{p}^{p}\right) \frac{\mathrm{d}}{\mathrm{d} t}\left\|\Delta^{m} w\right\|^{2}-M^{\prime}(\xi)\left(\left\|\nabla^{m} u_{1}\right\|_{p}^{p}-\left\|\nabla^{m} u_{2}\right\|_{p}^{p}\right)\left((-\Delta)^{2 m} u_{2}, w_{t}\right) \\
\geq \frac{\mu}{2} \frac{\mathrm{d}}{\mathrm{d} t}\left\|\Delta^{m} w\right\|^{2}-\frac{\beta}{2}\left\|\Delta^{m} w_{t}\right\|^{2}-\frac{C_{4}^{2}}{2 \beta \lambda_{1}^{m}}\left\|\Delta^{m} w\right\|^{2}, \\
\left|\left(\left|u_{1}\right|^{\rho} u_{1}-\left|u_{2}\right|^{\rho} u_{2}, w_{t}\right)\right| \geq-C_{5}\left|\int_{\Omega}\left(\left|u_{1}\right|^{\rho}+\left|u_{2}\right|^{\rho}\right)\right| w\left|\| w_{t}\right| \mathrm{d} x \mid \\
\geq-\frac{C_{6}}{2 \lambda_{1}^{2 m}}\left\|\Delta^{m} w\right\|^{2}-\frac{C_{6}}{2}\left\|w_{t}\right\|^{2} .
\end{gathered}
$$

Substituting (3.26)-(4.1) into (3.25), we obtain

$$
\frac{\mathrm{d}}{\mathrm{d} t}\left(\left\|w_{t}\right\|^{2}+\mu\left\|\Delta^{m} w\right\|^{2}\right) \leq\left|\beta \lambda_{1}^{2 m}-C_{6}\right|\left\|w_{t}\right\|^{2}+\left(\frac{C_{4}^{2}}{\beta \lambda_{1}^{m}}+\frac{C_{6}}{\lambda_{1}^{2 m}}\right)\left\|\Delta^{m} w\right\|^{2} .
$$

Let $\alpha_{3}=\max \left\{\left|\beta \lambda_{1}^{2 m}-C_{6}\right|, \frac{C_{4}^{2}}{\beta \mu \lambda_{1}^{m}}+\frac{C_{6}}{\mu \lambda_{1}^{2 m}}\right\}$, by using the integral Gronwall's inequality we receive that

$$
\left\|w_{t}(t)\right\|^{2}+\mu\left\|\Delta^{m} w(t)\right\|^{2} \leq\left(\left\|w_{t}(0)\right\|^{2}+\mu\left\|\Delta^{m} w(0)\right\|^{2}\right) \mathrm{e}^{\alpha_{3} t}=0 .
$$


Hence $w_{t}(t)=0, \Delta^{m} w(t)=0$, a.e. $w(t)=0, u_{1}=u_{2}$. The uniqueness of solution is proved.

\section{Global Attractor and Dimension Estimation}

Theorem 2. [9] Assume $E$ is a Banach space, and $\{S(t)\}_{t \geq 0}$ are the semigroup operators on $E . S(t): E \rightarrow E, S(t+s)=S(t) S(s)(\forall t, s \geq 0), \quad S(0)=I$, where $I$ is the unit operator. Suppose $S(t)$ satisfies the following conditions:

1) $S(t)$ is uniformly bounded, i.e. $\forall R>0,\|u\|_{E} \leq R$, there is a constant $C(R)$, such that

$$
\|S(t) u\|_{E} \leq C(R), \quad(\forall t \in[0,+\infty)) ;
$$

2) There is a bounded absorbing set $B_{0} \subset E$, i.e. $\forall B \subset E$, there exists a constant $t_{0}$, so that

$$
S(t) B \subset B_{0}, \quad\left(\forall t \geq t_{0}\right) ;
$$

3) As $t>0, S(t)$ is a completely continuous operator.

It is said that the semigroup operator $S(t)$ exists a compact global attractor $A$.

If $S(t)$ is the solution semigroup that generated by the initial boundary value problem (1.1), i.e. $u(t)=S(t) u_{0}$, according to Lemma 1 and Lemma 2 we obtain the existence theorem of the following family of global attractors.

Theorem 3. Under the assumption of Theorem 1, let $S(t)$ be the solution semigroup that generated by the initial boundary value problem (1.1), then the initial boundary value problem (1.1) exists a family of global attractors, i.e. exists compact set $A_{k} \subset E_{k} \subset E_{0}$, and

$$
A_{k}=\omega\left(B_{0 k}\right)=\bigcap_{s \geq 0} S(t \geq s) B_{0 k},
$$

where $B_{0 k}=\left\{(u, v) \in E_{k}:\|(u, v)\|_{E_{k}}^{2}=\|u\|_{H_{0}^{2 m+k}(\Omega)}^{2}+\|v\|_{H_{0}^{k}(\Omega)}^{2} \leq R_{k}^{2}\right\}, \quad B_{0 k} \quad$ is the bounded absorbing set in $E_{k}$ and satisfies:

1) $S(t) A_{k}=A_{k},(t>0)$;

2) $\lim _{t \rightarrow+\infty} \operatorname{dist}\left(S(t) B_{0 k}, A_{k}\right)=0$, where for arbitrary bounded set $B_{0 k} \subset E_{k}$, we have

$$
\operatorname{dist}\left(S(t) B_{0 k}, A_{k}\right)=\sup _{x \in B_{0 k}} \inf _{y \in A_{k}}\|S(t) x-y\|_{E_{k}} .
$$

Proof. The proof can be obtained by verifying the three conditions in Theorem 2. Under the conditions in Theorem 1, suppose that the Equation (1.1) has a solution semigroup $S(t): E_{k} \rightarrow E_{k}$.

(1)According to Lemma 2, for arbitrary $B_{0 k} \subset E_{k}$, it contains the bounded set of the sphere $\left\{\|(u, v)\|_{E_{k}} \leq R\right\}$ such that there exists a constant $C$, then we have

$$
\left\|S(t)\left(u_{0}, v_{0}\right)\right\|_{E_{k}}^{2}=\|u\|_{H_{0}^{2 m+k}(\Omega)}^{2}+\|v\|_{H_{0}^{k}(\Omega)}^{2} \leq R^{2}+C,
$$

where $t \geq 0,\left(u_{0}, v_{0}\right) \in B_{0 k},\{S(t)\}_{t \geq 0}$ is uniformly bounded in $E_{k}$.

2) Further, $\forall\left(u_{0}, v_{0}\right) \in E_{k}, t \geq \max \left(t_{1}, t_{2}\right)$, we have 


$$
\left\|S(t)\left(u_{0}, v_{0}\right)\right\|_{E_{k}}^{2}=\|u\|_{H_{0}^{2 m+k}(\Omega)}^{2}+\left\|v_{0}\right\|_{H_{0}^{k}(\Omega)}^{2} \leq R_{k}^{2}+R_{0}^{2},
$$

so $B_{0 k}$ is a bounded absorption set of the semigroup $S(t)$.

3) Due to $E_{k} \hookrightarrow E_{0}$, we obtain that the bounded set in $E_{k}$ is the compact set in $E_{0}$. Hence, $S(t)$ is completely continuous operator.

Theorem 3 is proved.

In order to estimate the Hausdorff dimension and Fractal dimension of the family of global attractor, we linearize the problem (1.1):

$$
\begin{aligned}
& U_{t t}+M\left(\left\|\nabla^{m} u\right\|_{p}^{p}\right)(-\Delta)^{2 m} U+M^{\prime}\left(\left\|\nabla^{m} u\right\|_{p}^{p}\right)\left(\left\|\nabla^{m} u\right\|_{p}^{p}\right)^{\prime} \nabla^{m} U(-\Delta)^{2 m} u \\
& +\beta(-\Delta)^{2 m} U_{t}+(\rho+1)|u|^{\rho} U=0, \\
& U(x, t)=0, x \in \partial \Omega, \quad t>0, \\
& U(x, 0)=\xi, U_{t}(x, 0)=\eta,
\end{aligned}
$$

where $(\xi, \eta) \in E_{k},\left(u, u_{t}\right) \in S(t)\left(u_{0}, u_{1}\right)$ is the solution of question (1.1) obtained by $\left(u_{0}, u_{1}\right) \in A_{k}$. Then for a given $\left(u_{0}, u_{1}\right), S(t): E_{k} \rightarrow E_{k}$, it can be proved that for arbitrary $(\xi, \eta) \in E_{k}$, there is a unique solution $\left(U(t), U_{t}(t)\right) \in L^{\infty}\left([0,+\infty) ; E_{k}\right)$ to the linear problem (4.1).

Lemma 4. For $\forall t>0, R>0$, the mapping $S(t): E_{k} \rightarrow E_{k}$ is differentiable on $E_{k}$. Then the derivative of $\varphi=\left(u_{0}, u_{1}\right)$ is a linear operator of $G(t):(\xi, \eta) \rightarrow\left(U(t), U_{t}(t)\right)$, where $\left(U(t), U_{t}(t)\right)$ is the solution of the linear initial boundary value problem (4.1).

Proof. Let $\varphi_{0}=\left(u_{0}, v_{0}\right) \in E_{k}, \widetilde{\varphi_{0}}=\left(u_{0}+\xi, v_{0}+\eta\right) \in E_{k}$, and $\left\|\varphi_{0}\right\|_{E_{k}} \leq R$, $\left\|\widetilde{\varphi_{0}}\right\|_{E_{k}} \leq R$, define $(u, v)=S(t) \varphi_{0},(\tilde{u}, \tilde{v})=S(t) \widetilde{\varphi_{0}}$, then $S(t)$ has Lipschitz property on the bounded set $E_{k}$ :

$$
\left\|S(t) \varphi_{0}-S(t) \widetilde{\varphi_{0}}\right\|_{E_{k}}^{2} \leq \mathrm{e}^{c t}\|(\xi, \eta)\|_{E_{k}}^{2} .
$$

Let $\Theta=\tilde{u}-u-U$ is the solution of the linear initial boundary value problem (4.1), then we have

$$
\begin{gathered}
\Theta_{t t}+M\left(\left\|\nabla^{m} u\right\|_{p}^{p}\right)(-\Delta)^{2 m} \Theta+\beta(-\Delta)^{2 m} \Theta_{t}=h_{1}+h_{2}, \\
\Theta(0)=\Theta_{t}(0)=0 . \\
h_{1}=\left(M\left(\left\|\nabla^{m} u\right\|_{p}^{p}\right)-M\left(\left\|\nabla^{m} \tilde{u}\right\|_{p}^{p}\right)\right)(-\Delta)^{2 m} \tilde{u} \\
+M^{\prime}\left(\left\|\nabla^{m} u\right\|_{p}^{p}\right)\left(\left\|\nabla^{m} u\right\|_{p}^{p}\right)^{\prime} \nabla^{m} U(-\Delta)^{2 m} u, \\
h_{2}=|u|^{\rho} u-|\tilde{u}|^{\rho} \tilde{u}+(\rho+1)|u|^{\rho} U
\end{gathered}
$$

Let $s=\nabla^{m} u, \quad \tilde{s}=\nabla^{m} \tilde{u}, \quad \bar{u}=\tilde{u}-u, \quad \xi_{1}=\left(1-\alpha_{4}\right) \tilde{s}+\alpha_{4} s$,

$\xi_{2}=\left(1-\alpha_{5}\right) \tilde{s}+\alpha_{5} \xi_{1}, \quad N(\xi)=M^{\prime}\left(\|s\|_{p}^{p}\right)\left(\|s\|_{p}^{p}\right)^{\prime}, \quad \alpha_{4}, \alpha_{5} \in(0,1)$.

Then 


$$
\begin{gathered}
h_{1}=-N\left(\xi_{1}\right) \nabla^{m} \bar{u}(-\Delta)^{2 m} \tilde{u}+N(s) \nabla^{m} \bar{u}(-\Delta)^{2 m} u-N(s) \nabla^{m} \Theta(-\Delta)^{2 m} u \\
=-N\left(\xi_{1}\right) \nabla^{m} \bar{u}(-\Delta)^{2 m} \bar{u}-N^{\prime}\left(\xi_{2}\right)\left(1-\alpha_{4}\right)\left(\nabla^{m} \bar{u}\right)^{2}(-\Delta)^{2 m} u \\
-N(s) \nabla^{m} \Theta(-\Delta)^{2 m} u \\
h_{2}=-(\rho+1)\left|\xi_{3}\right|^{\rho} \bar{u}+(\rho+1)|u|^{\rho} \bar{u}-(\rho+1)|u|^{\rho} \Theta \\
=\rho(\rho+1)\left|\xi_{4}\right|^{\rho-1} \bar{u}^{2}-(\rho+1)|u|^{\rho} \Theta .
\end{gathered}
$$

Taking the inner product of Equation (4.3) with $s=\nabla^{m} u$, we receive that

$$
\begin{aligned}
& \frac{1}{2} \frac{\mathrm{d}}{\mathrm{d} t}\left\|\nabla^{k} \Theta_{t}\right\|^{2}+\frac{1}{2} M\left(\left\|\nabla^{k} u\right\|_{p}^{p}\right) \frac{\mathrm{d}}{\mathrm{d} t}\left\|\nabla^{2 m+k} \Theta\right\|^{2}+\beta\left\|\nabla^{2 m+k} \Theta_{t}\right\|^{2} \\
& =\left(h_{1}+h_{2},(-\Delta)^{k} \Theta_{t}\right) \text {, } \\
& \left|\left(h_{1},(-\Delta)^{k} \Theta_{t}\right)\right| \leq\left\|N\left(\xi_{1}\right)\right\|\left\|_{\infty}\right\| \nabla^{2 m+k} \bar{u}\left\|\mid \Delta^{m} \bar{u}\right\|\left\|\nabla^{m+k} \Theta_{t}\right\| \\
& +C_{7}\left\|N^{\prime}\left(\xi_{2}\right)\right\|_{\infty}\left\|\nabla^{2 m+k} \bar{u}\right\|^{2}\left\|(-\Delta)^{2} u\right\|\left\|\Theta_{t}\right\| \\
& +\|N(s)\|_{\infty}\left\|(-\Delta)^{m} u\right\|\left\|\nabla^{m+k} \Theta\right\|\left\|\nabla^{k} \Theta_{t}\right\| \\
& \leq\left(\frac{C_{8}}{2 \lambda_{1}^{k}}+\frac{C_{10}}{2}\right)\left\|\nabla^{k} \Theta_{t}\right\|^{2}+\frac{C_{10}}{2 \lambda_{1}^{m}}\left\|\nabla^{2 m+k} \Theta\right\|^{2} \\
& +\frac{\beta}{2}\left\|\nabla^{2 m+k} \Theta_{t}\right\|^{2}+\left(\frac{C_{8}}{2}+\frac{C_{9}^{2}}{2 \beta \lambda_{1}^{m+k}}\right)\left\|\nabla^{2 m+k} \bar{u}\right\|^{4}, \\
& \left|\left(h_{2},(-\Delta)^{k} \Theta_{t}\right)\right| \\
& \leq \rho(\rho+1)\left\|\xi_{4}\right\|_{\infty}^{\rho-1}\left\|\nabla^{k} \bar{u}\right\|^{2}\left\|\Theta_{t}\right\|+(\rho+1)\|u\|_{\infty}^{\rho}\left\|\nabla^{k} \Theta\right\|\left\|\nabla^{k} \Theta_{t}\right\| \\
& =\frac{C_{11}}{2 \lambda_{1}^{4 m}}\left\|\nabla^{2 m+k} \bar{u}\right\|^{4}+\frac{C_{12}}{2 \lambda_{1}^{2 m}}\left\|\nabla^{2 m+k} \Theta\right\|^{2}+\left(\frac{C_{11}}{2 \lambda_{1}^{k}}+\frac{C_{12}}{2}\right)\left\|\nabla^{k} \Theta_{t}\right\|^{2} \text {. }
\end{aligned}
$$

According to (4.4)-(4.6), we obtain

$$
\begin{aligned}
& \frac{\mathrm{d}}{\mathrm{d} t}\left(\left\|\nabla^{k} \Theta_{t}\right\|^{2}+\mu\left\|\nabla^{2 m+k} \Theta\right\|^{2}\right)+\beta\left\|\nabla^{2 m+k} \Theta_{t}\right\|^{2} \\
& \leq\left(\frac{C_{8}+C_{11}}{\lambda_{1}^{k}}+C_{10}+C_{12}\right)\left\|\nabla^{k} \Theta_{t}\right\|^{2}+\left(\frac{C_{10}}{\lambda_{1}^{m}}+\frac{C_{12}}{\lambda_{1}^{2 m}}\right)\left\|\nabla^{2 m+k} \Theta\right\|^{2} \\
& +\left(C_{8}+\frac{C_{9}^{2}}{\beta \lambda_{1}^{m+k}}+\frac{C_{11}}{\lambda_{1}^{4 m}}\right)\left\|\nabla^{2 m+k} \bar{u}\right\|^{4} .
\end{aligned}
$$

Let $\alpha_{4}=\max \left\{\frac{C_{8}+C_{11}}{\lambda_{1}^{k}}+C_{10}+C_{12}, \frac{C_{10}}{\mu \lambda_{1}^{m}}+\frac{C_{12}}{\mu \lambda_{1}^{2 m}}\right\}$, we receive

$$
\frac{\mathrm{d}}{\mathrm{d} t}\left(\left\|\nabla^{k} \Theta_{t}\right\|^{2}+\mu\left\|\nabla^{2 m+k} \Theta\right\|^{2}\right) \leq \alpha_{4}\left(\left\|\nabla^{k} \Theta_{t}\right\|^{2}+\mu\left\|\nabla^{2 m+k} \Theta\right\|^{2}\right)+C_{13}\left\|\nabla^{2 m+k} \bar{u}\right\|^{4}
$$

By using the differential Gronwall's inequality, we have

$$
\left\|\nabla^{k} \Theta_{t}\right\|^{2}+\mu\left\|\nabla^{2 m+k} \Theta\right\|^{2} \leq C_{14} \mathrm{e}^{C_{15} t}\left\|(\xi, \eta)^{\mathrm{T}}\right\|_{E_{k}}^{4} .
$$

According to the Lipschitz property of $S(t)$, as $\left\|(\xi, \eta)^{\mathrm{T}}\right\|_{E_{k}}^{2} \rightarrow 0$, we obtain 


$$
\frac{\left\|S(t) \widetilde{\varphi_{0}}-S(t) \varphi_{0}-G(t)(\xi, \eta)\right\|_{E_{k}}^{2}}{\left\|(\xi, \eta)^{\mathrm{T}}\right\|_{E_{k}}^{2}} \leq C_{14} \mathrm{e}^{C_{15} t}\left\|(\xi, \eta)^{\mathrm{T}}\right\|_{E_{k}}^{2} \rightarrow 0 .
$$

That means $S(t)$ is uniformly differentiable on $E_{k}$.

Theorem 4. Under the conditions of Theorem 3, the family of global attractor $A_{k}$ of the initial boundary value problem (1.1) have the finite Hausdorff dimension and Fractal dimension, and $d_{H}\left(A_{k}\right)<\frac{N}{2}, d_{F}\left(A_{k}\right)<\frac{3 N}{2}$.

Proof. In order to estimate the dimension of the family of global attractor $A_{k}$, we rewrite the initial boundary value problem as a first-order evolution equation

$$
\Psi_{t}+\Lambda_{\varepsilon} \Psi+g(\Psi)=F(\Psi)
$$

where $\Psi=(u, v)^{\mathrm{T}}=\left(u, u_{t}+\varepsilon u\right)^{\mathrm{T}}, \quad g(\Psi)=\left(0,|u|^{\rho} u\right)^{\mathrm{T}}, \quad F(\Psi)=(0, f(x))^{\mathrm{T}}$,

$$
\Lambda_{\varepsilon}=\left(\begin{array}{cc}
\varepsilon I & -I \\
\left(M\left(\left\|\nabla^{m} u\right\|_{p}^{p}\right)-\varepsilon \beta\right)(-\Delta)^{2 m}+\varepsilon^{2} I & \beta(-\Delta)^{2 m}-\varepsilon I
\end{array}\right) .
$$

Further, let $L(\Psi)=\Psi_{t}=F(\Psi)-\Lambda_{\varepsilon} \Psi-g(\Psi)$, where $L: E_{k} \rightarrow E_{k}$ is Frechet differential.

Similarly, we rewrite the linear Equation (4.1) as

$$
P_{t}+\overline{\Lambda_{\varepsilon}} P+g^{\prime}(\phi) P=0,
$$

where $P=\left(U, U_{t}+\varepsilon U\right)^{\mathrm{T}}, \quad P_{t}=L_{t}(\Psi), \quad g^{\prime}(\phi) P=\left(0,(\rho+1)|u|^{\rho} U\right)^{\mathrm{T}}, \quad U$ is the solution of Equation (4.1),

$$
\begin{gathered}
\overline{\Lambda_{\varepsilon}}=\left(\begin{array}{cc} 
& -I \\
\left(M\left(\left\|\nabla^{m} u\right\|_{p}^{p}\right)-\varepsilon \beta\right)(-\Delta)^{2 m}+\varepsilon^{2} I+D & \beta(-\Delta)^{2 m}-\varepsilon I
\end{array}\right), \\
D \cdot U=M^{\prime}\left(\left\|\nabla^{m} u\right\|_{p}^{p}\right)\left(\left\|\nabla^{m} u\right\|_{p}^{p}\right)^{\prime} \nabla^{m} U(-\Delta)^{2 m} u .
\end{gathered}
$$

For every fixed $\left(u_{0}, v_{0}\right) \in E_{k}$, assume that $U_{1}(t), U_{2}(t), \cdots, U_{N}(t)$ are $N$ solutions to Equation (4.8). The initial value $U_{1}(0)=\xi_{1}, U_{2}(0)=\xi_{2}, \cdots, U_{N}(0)=\xi_{N}$, where $\xi_{i} \in E_{k}(i=1,2, \cdots, N)$.

Then

$$
\begin{aligned}
& \frac{\mathrm{d}}{\mathrm{d} t}\left\|U_{1}(t) \wedge U_{2}(t) \wedge \cdots \wedge U_{N}(t)\right\|_{\wedge E_{k}}^{2} \\
& -2 \operatorname{tr}\left(L_{t}(\Psi) \cdot Q_{N}(t)\right)\left\|\xi_{1} \wedge \xi_{2} \wedge \cdots \wedge \xi_{N}\right\|_{E_{k}}^{2}=0,
\end{aligned}
$$

where $\wedge$ represents the cross product, tr represents the trace of the operator, $Q_{N}$ represents the orthogonal projection from $E_{k}$ to $\operatorname{span}\left\{U_{1}(t), U_{2}(t), \cdots, U_{N}(t)\right\}$.

According to uniform Gronwall's inequality, we obtain 


$$
\begin{aligned}
& \left\|U_{1}(t) \wedge U_{2}(t) \wedge \cdots \wedge U_{N}(t)\right\|_{\wedge E_{k}}^{2} \\
& \leq\left\|\xi_{1} \wedge \xi_{2} \wedge \cdots \wedge \xi_{N}\right\|_{\wedge E_{k}}^{2} \exp \left(\int_{0}^{t} t r L_{t}(\Psi(\tau)) \cdot Q_{N}(\tau) \mathrm{d} \tau\right) .
\end{aligned}
$$

For arbitrarily given time $\tau$, let $\omega_{j}(\tau)=\left(\xi_{j}(\tau), \eta_{j}(\tau)\right)^{\mathrm{T}}(j=1,2, \cdots, N)$ are the orthogonal projection of $\operatorname{span}\left\{U_{1}(t), U_{2}(t), \cdots, U_{N}(t)\right\}$. Then, we have

$$
\left\|\nabla^{2 m+k} \xi_{j}\right\|^{2}+\left\|\nabla^{k} \eta_{j}\right\|^{2}=1 .
$$

Define the inner production on $E_{k}$ as $((\varsigma, \eta),(\bar{\varsigma}, \bar{\eta}))=\left(\nabla^{2 m+k} \varsigma, \nabla^{2 m+k} \bar{\varsigma}\right)+\left(\nabla^{k} \eta, \nabla^{k} \bar{\eta}\right)$.

According to the above conditions, we receive

$$
\begin{aligned}
& \operatorname{tr} L_{t}\left(\Psi(\tau) \cdot Q_{N}(\tau)\right)=\sum_{j=1}^{N}\left(L_{t}(\Psi(\tau)) \cdot Q_{N}(\tau) \omega_{j}(\tau), \omega_{j}(\tau)\right)_{E_{k}} \\
& =\sum_{j=1}^{N}\left(L_{t}(\Psi(\tau)) \omega_{j}(\tau), \omega_{j}(\tau)\right)_{E_{k}}, \\
& \text { where } \begin{aligned}
\left(L_{t}(\Psi(\tau)) \omega_{j}(\tau), \omega_{j}(\tau)\right)_{E_{k}} & =\left(L_{t}(\Psi(\tau)) \omega_{j}(\tau), \omega_{j}(\tau)\right)_{E_{k}} \\
& =-\left(\overline{\wedge_{\varepsilon}} \omega_{j}, \omega_{j}\right)-\left(g^{\prime}(\phi) \omega_{j}, \omega_{j}\right)
\end{aligned} \text {. } \\
& \left(\overline{\wedge_{\varepsilon}} \omega_{j}, \omega_{j}\right)=\left(\left(\varepsilon \xi_{j}-\eta_{j}, M\left(\left\|\nabla^{m} u\right\|_{p}^{p}\right)-\varepsilon \beta(-\Delta)^{2 m} \xi_{j}+\varepsilon^{2} \xi_{j}+D \cdot \xi_{j}\right.\right. \\
& \left.\left.+\beta(-\Delta)^{2 m} \eta_{j}-\varepsilon \eta_{j}\right),\left(\xi_{j}, \eta_{j}\right)\right) \\
& =\varepsilon\left\|\nabla^{2 m+k} \xi_{j}\right\|^{2}+\left(M\left(\left\|\nabla^{m} u\right\|_{p}^{p}\right)-\varepsilon \beta-1\right)\left(\nabla^{2 m+k} \xi_{j}, \nabla^{2 m+k} \eta_{j}\right) \\
& +\varepsilon^{2}\left(\nabla^{k} \xi_{j}, \nabla^{k} \eta_{j}\right)+\beta\left\|\nabla^{2 m+k} \eta_{j}\right\|^{2}-\varepsilon\left\|\nabla^{k} \xi_{j}\right\|^{2}+\left(\nabla^{k} D \cdot \xi_{j}, \nabla^{k} \eta_{j}\right) \\
& \geq\left(\varepsilon-\frac{\varepsilon^{2}(\beta-1)}{2}\right)\left\|\nabla^{2 m+k} \xi_{j}\right\|^{2}+\frac{(\beta+1) \lambda_{1}^{2 m}-\varepsilon^{2}-C_{16}}{2}\left\|\nabla^{k} \eta_{j}\right\|^{2} \\
& -\frac{\varepsilon^{2}+2 \varepsilon+C_{16}}{2}\left\|\nabla^{k} \xi_{j}\right\|^{2} \\
& \left(g^{\prime}(\phi) \omega_{j}, \omega_{j}\right)=\left(\left(0, \rho|u|^{\rho} \eta_{j}\right),\left(\xi_{j}, \eta_{j}\right)\right) \\
& \geq-\rho\|u\|_{\infty}^{\rho}\left\|\nabla^{k} \eta_{j}\right\|^{2} \geq-C_{17}\left\|\nabla^{k} \eta_{j}\right\|^{2} .
\end{aligned}
$$

According to (4.10)-(4.13), let

$\delta=\min \left\{\varepsilon-\frac{\varepsilon^{2}(\beta-1)}{2}, \frac{(\beta+1) \lambda_{1}^{2 m}-\varepsilon^{2}-C_{16}}{2}-C_{17}\right\}, \quad \gamma=\frac{\varepsilon^{2}+2 \varepsilon+C_{16}}{2}$, we obtain

$$
\sum_{j=1}^{N}\left(L_{t}(\Psi(\tau)) \omega_{j}(\tau), \omega_{j}(\tau)\right)_{E_{k}} \leq-N \delta+\gamma \sum_{j=1}^{N}\left\|\nabla^{k} \xi_{j}\right\|^{2} .
$$

For almost all $t$, we have

$$
\sum_{j=1}^{N}\left\|\nabla^{k} \xi_{j}\right\|^{2} \leq \sum_{j=1}^{N} \lambda_{j}^{-\frac{2 m-1}{2 m}}
$$


where $\lambda_{j}$ is the eigenvalue of $(-\Delta)^{2 m}$. Thus

$$
\operatorname{tr}\left(L_{t}(\Psi)(\tau) \cdot Q_{N}(\tau)\right) \leq-N \delta+\gamma \sum_{j=1}^{N} \lambda_{j}^{-\frac{2 m-1}{2 m}} .
$$

Suppose

$$
\begin{gathered}
q_{N}(t)=\sup _{\Psi_{0} \in A} \inf _{\xi_{j} \in E_{k}}\left(\frac{1}{t} \int_{0}^{t} \operatorname{tr} L_{t}\left(S(\tau) \Psi_{0}\right) \cdot Q_{N}(\tau) \mathrm{d} \tau\right), \\
q_{N}=\lim _{t \rightarrow \infty} q_{N}(t) .
\end{gathered}
$$

Due to (4.14), we have

$$
q_{N} \leq-N \delta+\gamma \sum_{j=1}^{N} \lambda_{j}^{-\frac{2 m-1}{2 m}}
$$

Therefore, the Lyapunov exponents $\sigma_{1}, \sigma_{2}, \cdots, \sigma_{N}$ of $B_{0 k}$ are uniformly bounded, moreover

$$
\sigma_{1}+\sigma_{2}+\cdots+\sigma_{N} \leq-N \delta+\gamma \sum_{j=1}^{n} \lambda_{j}^{-\frac{2 m-1}{2 m}}
$$

such that

$$
\begin{gathered}
\left(q_{j}\right)_{+} \leq-N \delta+\gamma \sum_{j=1}^{n} \lambda_{j}^{\frac{2 m-1}{2 m}} \leq \gamma \sum_{j=1}^{N} \lambda_{j}^{\frac{2 m-1}{2 m}} \leq-\frac{N \delta}{3} \\
q_{N} \leq-N \delta\left(1-\frac{\gamma}{N \delta} \sum_{j=1}^{N} \lambda_{j}^{\frac{2 m-1}{2 m}}\right) \leq-\frac{2 N \delta}{3} .
\end{gathered}
$$

Further,

$$
\max _{1 \leq j \leq N} \frac{\left(q_{j}\right)_{+}}{\left|q_{N}\right|} \leq \frac{1}{2} .
$$

Thus, we obtain $d_{H}\left(A_{k}\right)<\frac{N}{2}, d_{F}\left(A_{k}\right)<\frac{3 N}{2}$.

\section{Conflicts of Interest}

The authors declare no conflicts of interest regarding the publication of this paper.

\section{References}

[1] Kirchhoff, G. (1883) Vorlesungen fiber Mechanik. Teubner, Stuttgart.

[2] Hosoya, M. and Yamada, Y. (1991) On Some Nonlinear Wave Equations II: Global Existence and Energy Decay of Solutions. Journal of the Faculty of Science, the University of Tokyo. Section 1A, Mathematics, 38, 239-250.

[3] Chueshov, I. (2011) Long-Time Dynamics of Kirchhoff Wave Models with Strong Nonlinear Damping. Journal of Differential Equations, 252, 1229-1262. https://doi.org/10.1016/j.jde.2011.08.022

[4] Lin, G.G. and Chen, Y.H. (2020) A Family of Global Attractors for the Higher-Order Kirchhoff-Type Equations and Its Dimension Estimation. American Journal of Applied Mathematics, 8, 300-310. 
[5] Lin, G.G. and Guan, L.P. (2019) Global Attractor Family and Its Dimension Estimation of Higher-Order Kirchhoff Type Equations with Strong Damping. Acta Analysis Functionalis Applicata, 21, 268-281.

[6] Lin, G.G. and Wang, W. (2018) Exponential Attractors for the Higher-Order Kirchhoff-Type Equation with Nonlinear Damped Term. Acta Analysis Functionalis Applicata, 20, 19-25.

[7] Li, Q.X. and Tan, Z. (2002) Global Existence of a Kirchhoff Type Equation with Damping and Source Terms. Journal of Xiamen University (Natural Science), No. 4, 418-422.

[8] Lin, G.G. and Li, Z.X. (2019) Attractor Family and Dimension for a Class of High-Order Nonlinear Kiechhoff Equations. Journal of Shandong University (Natural Science), 54, 1-11.

[9] Lin, G.G. (2011) Nonlinear Evolution Equations. Yunnan University Press, Kunming.

[10] Wang, Y.H. and Zhong, C.K. (2013) Upper Semicontinuity of Pullback Attractors for Nonautonomous Kirchhoff Wave Models. Discrete \& Continuous Dynamical Systems, 33, 3189-3209. https://doi.org/10.3934/dcds.2013.33.3189

[11] Zhang, S.L., Zhang, J.W. and Wang, H.Y. (2020) Global Attractors for Kirchhoff Wave Equation with Nonlinear Damping and Memory. Boundary Value Problems, 2020, Article No. 116. https://doi.org/10.1186/s13661-020-01413-5

[12] Ye, Y.J. and Tao, X.X. (2019) Initial Boundary Value Problem for Higher-Order Nonlinear Kirchhoff-Type Equation. Acta Mathematica Sinica, Chinese Series, 62, 923-938.

[13] Gao, Y.L., Sun, Y.T. and Lin, G.G. (2016) The Global Attractor for the Higher-Order Kirchhoff-Type Equation with Nonlinear Strongly Damped Term. International Journal of Modern Nonlinear Theory and Application, 10, 6608-6621.

[14] Lin, G.G. and Yang, S.M. (2017) The Global Attractors for the Higher-Order Coupled Kirchhoff-Type Equation with Strongly Damped Terms and Source Term. European Journal of Mathematics and Computer Science, 4, 66-78.

[15] Yang, Z.J., Ding, D.Y. and Li, L. (2016) Longtime Dynamics of the Kirchhoff Equations with Fractional Damping and Supercritical Nonlinearity. Journal of Mathematical Analysis and Applications, 422, 485-510. https://doi.org/10.1016/j.jmaa.2016.04.079

[16] Teman (1988) Infinite Dimensional Dynamical Systems in Mechanics and Physics. Springer-Verlag, New York, 15-26. https://doi.org/10.1007/978-1-4684-0313-8 